\title{
Obstetric and perinatal outcomes of dizygotic twin pregnancies resulting from In Vitro Fertilization versus spontaneous conception: a retrospective study
}

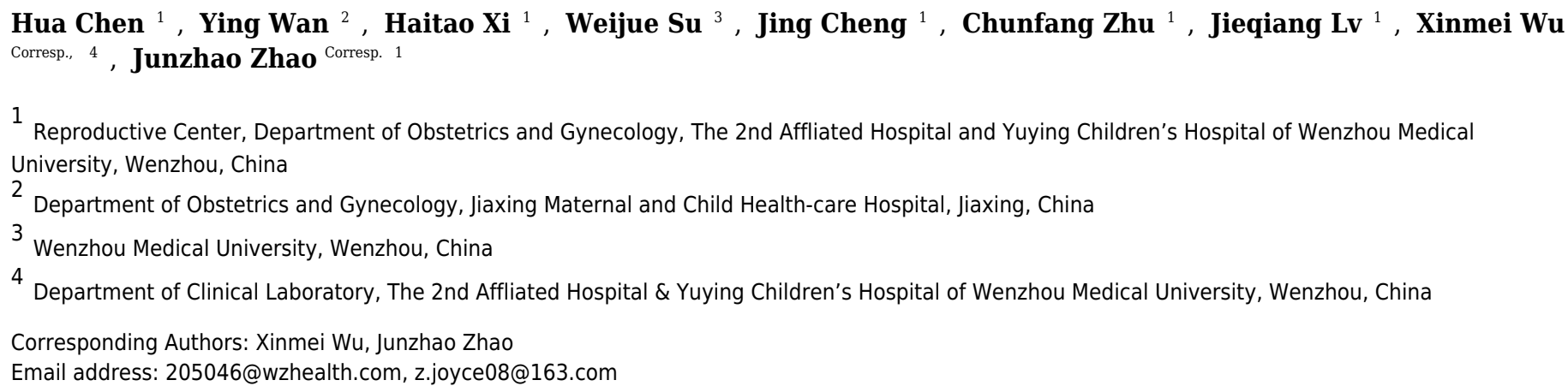

This study was designed to to assess perinatal and neonatal outcomes of dizygotic twin pregnancies conceived naturally or by IVF. After strict selection, the study included $\mathbf{4 7 0}$ dizygotic twin pregnancies. There were 249 resulting from IVF treatments and 221 conceiving spontaneously. After adjusting maternal age and primiparity, the results showed that there were no significant differences between the two groups $(P>0.05)$ in terms of maternal antenatal complications and neonatal outcomes. In conclusion, our study does not reveal increased risks for pregnancy-related complications and adverse neonatal outcomes in dizygotic twin pregnancies following IVF treatments. With these fundamental data, this study could provide a reference for perinatal care and clinical ART treatment and help to inform infertile parents about the potential risks of IVF treatments. 
1 Obstetric and perinatal outcomes of dizygotic twin pregnancies resulting from

2 In Vitro Fertilization versus spontaneous conception: a retrospective study

3

4 Hua Chen ${ }^{1}$, Ying $\mathrm{Wan}^{2}$, Haitao $\mathrm{Xi}^{1}$, Weijue $\mathrm{Su}^{3}$, Jing Cheng ${ }^{1}$, Chunfang $\mathrm{Zhu}^{1}$, Jieqiang $\mathrm{Lv}^{1}$,

5 Xinmei $\mathrm{Wu}^{4}$, Junzhao Zhao ${ }^{1}$

6 Affiliations of authors:

$7{ }^{1}$ Reproductive Center, Department of Obstetrics and Gynecology, The 2nd Affliated Hospital

8 and Yuying Children's Hospital of Wenzhou Medical University, Wenzhou, China

92 Department of Obstetrics and Gynecology, Jiaxing Maternal and Child Health-care Hospital,

10 Jiaxing, China

$11{ }^{3}$ Wenzhou Medical University, Wenzhou, China

${ }^{4}$ Department of Clinical Laboratory, The 2nd Affliated Hospital \& Yuying Children's Hospital of Wenzhou Medical University, Wenzhou, China

corresponding author: Xinmei Wu and Junzhao Zhao

E-mail: 205046@126.com, z.joyce08@163.com 
Abstract:

This study was designed to to assess perinatal and neonatal outcomes of dizygotic twin pregnancies conceived naturally or by IVF. After strict selection, the study included 470 dizygotic twin pregnancies. There were 249 resulting from IVF treatments and 221 conceiving spontaneously. After adjusting maternal age and primiparity, the results showed that there were no significant differences between the two groups $(P>0.05)$ in terms of maternal antenatal complications and neonatal outcomes. In conclusion, our study does not reveal increased risks for pregnancy-related complications and adverse neonatal outcomes in dizygotic twin pregnancies following IVF treatments. With these fundamental data, this study could provide a reference for perinatal care and clinical ART treatment and help to inform infertile parents about the potential risks of IVF treatments. 


\section{Introduction}

The rapid progress and wide application of Assisted Reproductive Technology (ART), especially in vitro fertilization and embryo transfer (IVF-ET), make it possible for infertile couples to conceive a baby successfully. Unlike spontaneous conception, however, to maximize the possibility of obtaining a live birth, two or more embryos are routinely transferred into the uterus (Vasario et al., 2010). Consequently, the incidence of multiple gestation rapidly rises (Farhi et al., 2013), which in turn raises perinatal and neonatal risks (Qin et al., 2015).

In spontaneous-conceived (SC) pregnancy, the incidence of multiple gestation is about $2 \%$, while this rate is up to $40 \%-50 \%$ in IVF-conceived pregnancies (Reynolds et al., 2003). Notably, twin pregnancy accounts for the majority of multiple gestations. Although single embryo transfer has been suggested, however, the implementation of single embryo transfer may reduce success rate. So how to successfully carrying it out without compromising the clinical outcomes is yet a technological challenge. To date, the strategy of at least two embryo for transfer is routinely performed in most reproductive centers.

Actually, twin pregnancies are known to be related to higher risks of maternal and fetal complications than singleton pregnancies in natural conception (Qin et al., 2015; Rao et al., 2004), it is yet unclear whether twin pregnancies following IVF treatments are associated with higher risks of obstetric and neonatal conditions when compared with SC twin pregnancies. Several studies have been performed to explore the exact role of IVF-ET in obstetric and neonatal outcomes of twin pregnancies, but the results remain conflicting (Geisler et al., 2014; Helmerhorst et al., 2007; McDonald et al., 2005; Pourali et al., 2016; Qin et al., 2015; Sun et al., 2016; Vasario et al., 2010). One of the confounding factors in some studies is that monochorionicity among IVF-conceived twin pregnancies is quite rare, when compared with that in SC twin pregnancies (about 2\% and 22\%, respectively), and monochorionic pregnancies have more adverse effects on obstetric outcomes (Penava et al., 2004). As a result, the adverse impact of IVF treatments on the outcomes of IVF-conceived twins may be compensated by the lower incidence of monochorionicity. Accordingly, it is necessary to exclude monozygotic twins and merely consider dizygotic twins. In addition, other factors responsible for the conflicting outcomes can be ascribed to the differences in study populations and the management approaches in twin pregnancies related studies.

Given current evidence on this topic is contradictory, and the results in medical literatures 
might vary greatly due to study populations, management approaches, and sample size. This study is thus designed to assess perinatal and neonatal outcomes of dizygotic twin pregnancies conceived naturally or by IVF.

\section{Materials and Methods}

This retrospective study was performed at the Second Affiliated Hospital of Wenzhou Medical University during the period from July 2015 to April 2016, and was approved by the Ethics Committee of the hospital. Informed consent was not needed for this retrospective investigation. The subjects in this study were mainly from Wenzhou area and its surrounding area, South-East Zhejiang province, China.

Patients who had antenatal care and delivered at Obstetric department of the Second Affiliated Hospital of Wenzhou Medical University during the period from July 2015 to April 2016 were taken into consideration. Only dizygotic twin pregnancies delivered after 28 weeks' gestation were included. In the IVF-conceived group, only those conceived following IVF/ICSI treatments were included, those conceived by other forms of Assisted Reproduction Technology were excluded. Twin gestations obtained after natural abortion or fetal reduction in multiple pregnancies were excluded. After selection, 470 patients were included, 249 dizygotic pregnancies conceived by IVF and 221 conceived spontaneously, as described in Figure 1.

Data were obtained from medical records, which include maternal age, gestational age, delivery mode, duration of hospitalization, perinatal complications, neonatal birth defects, birthweight, and neonatal intensive care unit (NICU) admission. The medical diagnosis was categorized according to the criteria proposed by Sun et al. (Sun et al., 2016).

The diagnosis of dichorionic twin pregnancies was based on ultrasounds between 6 and 10 weeks' gestation by the observation of two gestational sacs on vaginal sonography in the first trimester. Chorionicity was confirmed by examination of the placenta following delivery. Gestational age was calculated from 14 days before the embryo transfer for IVF-conceived women, and from the first day of the last menstrual cycle for SC women. Length of hospitalization was calculated from the day of admission to the day of discharge after delivery.

Gestational hypertension is the development of new hypertension in a pregnant woman after 20 weeks' gestation without the presence of protein in the urine or other signs of preeclampsia. Hypertension is defined as having a blood pressure greater than 140/90 mmHg. GDM was diagnosed by oral $75 \mathrm{~g}$ glucose tolerance test (OGTT) at between24 and 28 weeks' gestation. 
109 According to the International association for research on gestational diabetes 2010. The glucose 110 threshold of diagnosing GDM is $5.1 \mathrm{mmol} / \mathrm{L}$ for fasting blood test, $10.0 \mathrm{mmol} / \mathrm{L}$ at 1 hour after 111 OGTT, $8.5 \mathrm{mmol} / \mathrm{L}$ at 2 hours after OGTT. Premature rupture of membranes was documented as 112 rupture of membranes prior to the woman goes into labor. Maternal anemia was determined as a 113 decrease in whole-blood hemoglobin concentration of more than standard deviations below the mean of an age-matched reference range. Placenta previa was recorded as after 28 weeks of gestation, the placenta attaches to the lower segment of the uterus, and even the lower margin of the placenta reaches or covers the cervical orifice, and its position is lower than the first exposed part of the fetus. Placental acrete is defined as the abnormal adherence to or invasion of the villous tissue into the myometrium secondary to damage to the endometrium-myometrial interface of the uterine wall. Postpartum hemorrhage was defined as vaginal blood loss of $\geq 500$ $\mathrm{ml}$ within 24 hours after delivery, and when blood loss during cesarean delivery exceeds $1000 \mathrm{ml}$. Intrahepatic cholestasis of pregnancies was based on: 1) onset of generalized pruritus in the second or third trimester of pregnancy; 2) bile acid level $>10 \mu \mathrm{mol} / \mathrm{L}$; and 3) spontaneous relief within 3 weeks after delivery. The standard of diagnosing polyhydramnios by B-ultrasound is amniotic fluid index (AFI) $\geq 25 \mathrm{~cm}$ or amniotic fluid volume (AFV) $\geq 8 \mathrm{~cm}$. Placental abruption is defined as after 20 weeks of gestation or during childbirth, the placenta in its normal position is partially or completely removed from the wall of the uterus before the fetus is delivered. classified according to Hjalmarson (Hjalmarson O, 1981). Transient tachypnea, respiratory distress, and other types of respiratory disturbances were included in the analysis. Stillbirth is the death of a fetus in the uterine cavity after 20 weeks of gestation. Malformation was mainly defined whether the defecti was fatal or potentially life-threatening or likely to lead to serious handicap or major cosmetic defect if not surgically corrected. As to the rate of stillbirth and malformation, the numerator is the number of stillbirth and malformation, while the denominator is the number of twin deliveries. NICU admission and birth weight discordance (BWD) $>25 \%$ calculated by the following formula: ((birth weight of larger twin-birth weight of smaller twin)/ birth weight of larger twin) $\times 100 \%$.

Statistical analysis was performed using the Statistical Package for the Social Sciences, 
140

141

142

143

144

145

146

147

148

149

150

151

152

153

154

155

156

157

158

159

160

161

162

163

164

165

166

167

168

169

170

version 22.0 (SPSS Inc., Chicago, IL). Differences between groups were tested statistically using the Chi-square test for categorical data and the independent-sample $t$-test for continuous variables. A multiple logistic regression model was established to assess the association between the selected variables and the chance of pregnancy complications. $P<0.05$ was considered statistically significant.

\section{Results}

The maternal age and the proportion of primiparity in the IVF-conceived group were significantly higher than in the SC group $(30.66 \pm 3.80$ versus $28.44 \pm 4.27$ years old, $P<0.05$, and $85.5 \%$ versus $62.4 \%, P<0.05$, respectively). The average duration of hospitalization in IVFconceived population was significantly longer than in SC women $(7.34 \pm 4.99$ versus $6.98 \pm 4.92$ days, $P<0.05)$. In contrast, the proportion of cesarean section and were similar $(96 \%$ versus $93.2 \%, P>0.05)$ in both groups. When the comparison of first cesarean section between the two groups was restricted to women with at least one previous delivery, the proportion of first cesarean section were similar $(27.8 \%$ versus $29.3 \%, P>0.05)$. The average gestational age in the SC group was $36.41 \pm 1.65$ weeks, which was significantly longer than $36.07 \pm 1.71$ weeks in the IVF-conceived group (Table 1). The birth weight between the two groups were similar (2490.57 $\pm 432.37 \mathrm{~kg}$ versus $2484.05 \pm 428.03 \mathrm{~kg}, P>0.05)$.

After adjustment for maternal age and primiparity, regression analysis revealed there were no significant differences between the two groups $(P>0.05)$ in terms of maternal complications, with one exception of anemia ( $P=0.05$ ), as described in Table 2 .

Neonatal outcomes are shown in Table 3. There were no significant differences in terms of the incidence of low birth weight, growth discordance, neonatal asphyxia and admission for NICU $(P>0.05)$. The stillbirth and malformation were described in Table 4, two cases in two different twins were included in SC group. Specifically, one is intrauterine fetal death infant, and the other is cystic mass of chest wall infant. In the IVF group there were four stillbirths and three malformed infants, including four intrauterine fetal death infants, two congenital heart malformation infants and one congenital high jejunal atresia infant. Moreover, the seven cases came from seven different pairs. However, statistical analysis was not performed on stillbirth and malformation due to the small number and rare conditions. 
171

172

173

174

175

176

177

178

179

180

181

182

183

184

185

186

187

188

189

190

191

192

193

194

195

196

197

198

199

200

201

\section{Discussion}

In the past few years, growing interests has been paid to the role of IVF-ET treatments in obstetric and neonatal outcomes of twin pregnancies, but the results remain to be inconsistent (Geisler et al., 2014; Helmerhorst et al., 2007; McDonald et al., 2005; Moini et al., 2012; Pourali et al., 2016; Qin et al., 2015; Sun et al., 2016; Vasario et al., 2010). The current study compared the obstetric complications and neonatal outcomes of dizygotic twin gestations conceived via IVF with those from SC twin pregnancies.

During the past two decades, the rate of cesarean section in China mainland has risen rapidly. Elective and emergency caesarean section may contribute to this situation (Geisler et al., 2014). Moreover, there is no exception for the twin pregnancy population. Consistent with the study by Moini et al. (Moini et al., 2012), the rate of caesarean deliveries in the present study was similarly high in both twin pregnancy groups. Twin pregnancy has been known to be associated with increased morbidity and mortality for the mother and neonate (Rao et al., 2004). This intrinsic risk may be responsible for the high prevalence of cesarean section in this study. In contrast, some studies have shown similar, but lower rates of caesarean delivery. Lambalk et al. reported a much lower rate of caesarean delivery (30\% and 37\%) in both twin gestations groups (Lambalk et al., 2001). However, several other studies have demonstrated higher rates of caesarean delivery among IVF-conceived twin pregnancies (Domingues et al., 2014; Geisler et al., 2014; Helmerhorst et al., 2007; Pourali et al., 2016; Vasario et al., 2010). These results indicate that differences in study population and methodology for twin pregnancies related studies likely lead to the conflicting results.

In this study, the gestational age in the IVF-conceived group was significantly shorter than that in the SC group. However, the gap between the two groups was as small as a difference of 0.34 weeks, which means a little less than two and a half days. Meanwhile, $2 \sim 5$ days' in vitro culture time before embryo transfer should also be added into the patients' pregnancy duration of IVF-conceived group. Accordingly, the difference between the two groups may be meaningless though it is statistically significant.

Actually, the difference in gestational time is the indication of the incidence of prematurity. Significantly shorter gestational time in IVF-conceived population has been shown in similar studies done by Nassar (Nassar et al., 2003), Kallen (Kallen et al., 2010) and Caserta (Caserta et al., 2014). On one hand, this condition may be attributable to the infertility history and the 
202 importance of childbirth in the IVF group, obstetricians hence prefer to perform cesarean section 203 ahead of schedule (Pourali et al., 2016; Vannuccini et al., 2016). On the other hand, families 204 facing infertility usually believe that a trial of vaginal birth is more risky than cesarean section. Therefore, pregnant women are more inclined to choose cesarean section earlier than the expected date of childbirth.

Several previous studies have demonstrated that, in contrast to natural singleton pregnancy, IVF-conceived singleton gestation is independently associated with increased obstetric complications (Farhi et al., 2013; Pinborg et al., 2013). But does this effect also occurs in twin pregnancies when compared with SC twin pregnancies? The findings are conflicting. Some studies have shown that IVF treatments in dizygotic twin gestations are related to increased risks of obstetric complications, specifically preeclampsia, intrahepatic cholestasis of pregnancies (ICP) (Sun et al., 2016), and GDM (Moini et al., 2012; Pourali et al., 2016). In contrast, this relationship was not observed in several other studies (Anbazhagan et al., 2014; Geisler et al., 2014; Moini et al., 2012; Vasario et al., 2010). In addition, a systematic review demonstrated that perinatal mortality in twins conceived via ART was $40 \%$ lower compared with SC twins (Helmerhorst et al., 2004). When comparing the obstetric outcomes of IVF-conceived dichorionic twin pregnancies with those of SC dizygotic twin pregnancies, this study showed similar incidences of maternal and perinatal complications in terms of gestational diabetes mellitus (GDM), hypertensive disorder, premature rupture of membranes, maternal anemia, placenta previa, placental accrete, postpartum hemorrhage, drug use, ICP, and polyhydramnios. The only exception was maternal anemia, which was more frequently observed in the IVF twins group. However, the marginal $P$ value $(p=0.05)$ is inadequate to allow valid estimation of the real prevalence of maternal anemia in the present study population, and the exact conclusion warrants further assessment based on larger sample size.

It has been suggested that some infertility factors and IVF treatment itself may lead to the increased risks of perinatal complications. Unfortunately, because of the inadequate information obtained during the primary study, we could not address the effects of different infertility factors on perinatal outcomes. Thereby, our study still leaves open the question of whether one or a combination of infertility factors results in adverse perinatal outcomes.

With regard to the neonatal outcomes of twin pregnancies from IVF and natural conception, the literature provides contradictory findings. One meta-analysis (Hansen et al., 2013) and one 
233 well-designed study (Qin et al., 2015) concluded that multiple pregnancies resulting from IVF-

234 ET were at significantly greater risks for adverse pregnancy outcomes than SC multiple

235 pregnancies. Similarly, Saccone et al. performed a study including 668 women and showed that

236 IVF-conceived oligohydramnios pregnancies had a higher rate of spontaneous preterm birth than

237 SC dichorionic pregnancies (Saccone et al., 2017). It is hypothesized that underlying infertility

238 factors and invasive IVF-ET manipulations contribute to this condition (Hansen et al., 2013;

239 Nassar et al., 2003; Saccone et al., 2017). However, in the current study, IVF treatments did not

240 show any adverse effects on neonatal outcomes. The conclusion was in agreement with that in

241 several previous studies, which also have failed to find the increased risks when comparing the

242 neonatal outcomes according to mode of conception (Anbazhagan et al., 2014; Pourali et al.,

243 2016; Sun et al., 2016; Vasario et al., 2010). Differences in study population and methodology

244 may account for the conflicting data.

245 Although the present study used strict inclusion criteria, limitations do exist. First, some

246 patients conceived by ovulation induction and artificial insemination may have been categorized

247 into the SC group, and it has been reported that negative perinatal outcomes are more frequently

248 observed in multiple pregnancies resulting from induction and artificial insemination compared

249 with those conceived naturally (Qin et al., 2015). This may underestimate the relationship

250 between IVF-ET and adverse outcomes. Second, the retrospective nature of data collection and

251 the single-center study may restrict the strength and the quality of evidence. A well-controlled,

252 multicenter study may be more convincing to address the issue. In addition, low number of cases

253 may also lead to insufficient power of this kind of study.

254 Conclusion

255 Although older age and shorter gestational age were more frequent in the IVF-conceived twin

256 pregnancy population, this study did not show any increased risks for pregnancy-related

257 complications and adverse neonatal outcomes in dizygotic twin pregnancies following IVF. With

258 these fundamental data, this study could provide a reference for perinatal care and clinical ART

259 treatment and help to inform infertile parents about the potential risks of IVF.

260 Acknowledgements

261 We would like thank all personnel of the Reproductive Center, Department of OB \& GYN, the

262 2nd Affiliated Hospital \& Yuying Children's Hospital of Wenzhou Medical University, for their 263 assistance in this work. 
264

265

266

267

268

269

270

271

272

273

274

275

276

277

278

279

280

281

282

283

284

285

286

287

288

289

290

291

292

293

294

\section{Reference}

Anbazhagan A, Hunter A, Breathnach FM, Mcauliffe FM, Geary MP, Daly S, Higgins JR, Morrison JJ, Burke G, Higgins S, Dicker P, Tully E, Carroll S, Malone FD. 2014. Comparison of outcomes of twins conceived spontaneously and by artificial reproductive therapy. J Matern Fetal Neonatal Med 27:458-462.

Caserta D, Bordi G, Stegagno M, Filippini F, Podagrosi M, Roselli D, Moscarini M. 2014. Maternal and perinatal outcomes in spontaneous versus assisted conception twin pregnancies. European Journal of Obstetrics \& Gynecology and Reproductive Biology 174:64-69.

Domingues AP, Dinis SR, Belo A, Couto D, Fonseca E, Moura P. 2014. Impact of induced pregnancies in the obstetrical outcome of twin pregnancies. Fertility and Sterility 101:172177.

Farhi A, Reichman B, Boyko V, Hourvitz A, Ron-EI R, Lerner-Geva L. 2013. Maternal and neonatal health outcomes following assisted reproduction. Reproductive Biomedicine Online 26:454-461.

Geisler ME, O'Mahony A, Meaney S, Waterstone JJ, O'Donoghue K. 2014. Obstetric and perinatal outcomes of twin pregnancies conceived following IVF/ICSI treatment compared with spontaneously conceived twin pregnancies. Eur J Obstet Gynecol 33:1-6.

Hansen M, Kurinczuk JJ, Milne E, de Klerk N, Bower C. 2013. Assisted reproductive technology and birth defects: a systematic review and meta-analysis. Human Reproduction Update 19:330-353.

Helmerhorst FM, Perquin DA, Donker D, Keirse MJ. 2004. Perinatal outcome of singletons and twins after assisted conception: a systematic review of controlled studies. BMJ 328:261-265.

Hjalmarson O. 1981. Epidemiology and classification of acute, neonatal respiratory disorders: a prospective study. Acta Paediatr Scand 70:773-783

Jacquemy Y, Martens E, Martens G. 2014. Pregnancy at late premenopausal age: outcome of pregnancies at 45 years and older in Flanders, Belgium. J Obstet Gynaecol 34:479-481.

Kallen B, Finnstrom O, Lindam A, Nilsson E, Nygren K-G, Otterblad Olausson P. 2010. Selected neonatal outcomes in dizygotic twins after IVF versus non-IVF pregnancies. BJOG 117:676-682.

Lambalk CB, van Hooff M. 2001. Natural versus induced twinning and pregnancy outcome: a 
Dutch nationwide survey of primiparous dizygotic twin deliveries. Fertility and Sterility 75:731-736.

McDonald S, Murphy K, Beyene J, Ohlsson A. 2005. Perinatal outcomes of in vitro fertilization twins: a systematic review and meta-analyses. Am J Obstet Gynecol. 193:141152.

Moini A, Shiva M, Arabipoor A, Hosseini R, Chehrazi M, Sadeghi M. 2012. Obstetric and neonatal outcomes of twin pregnancies conceived by assisted reproductive technology compared with twin pregnancies conceived spontaneously: a prospective follow-up study. European Journal of Obstetrics \& Gynecology and Reproductive Biology 165:29-32.

Nassar AH, Usta IM, Rechdan JB, Harb TS, Adra AM, Abu-Musa AA. 2003. Pregnancy outcome in spontaneous twins versus twins who were conceived through in vitro fertilization. Am J Obstet Gynecol 189:513-518.

Penava, D, Natale, R. 2004. An association of chorionicity with preterm twin birth. J Obstet Gynaecol Canada 26:571-574.

Pinborg A, Wennerholm UB, Romundstad LB, Loft A, Aittomaki K, Söderström-Anttila V, Nygren KG, Hazekamp J, Berqh C. 2013. Why do singletons conceived after assisted reproduction technology have adverse perinatal outcome? Systematic review and metaanalysis. Human Reproduction Update 19:87-104.

Pourali L, Ayati S, Jelodar S, Zarifian A, Sheikh Andalibi MS. 2016. Obstetrics and perinatal outcomes of dichorionic twin pregnancy following ART compared with spontaneous pregnancy. Int J Reprod Biomed (Yazd) 14:317-322.

Qin JB, Wang H, Sheng XQ, Liang DS, Tan HZ, Xia JH. 2015. Pregnancy-related complications and adverse pregnancy outcomes in multiple pregnancies resulting from assisted reproductive technology: a meta-analysis of cohort studies. Fertility and Sterility 103:1492-508.

Rao A, Sairam S, Shehata H. 2004. Obstetric complications of twin pregnancies. Best Pract Res Clin Obstet Gynaecol 18:557-576.

Rebarber A, Istwan NB, Russo-Stieglitz K, Cleary-Goldman J, Rhea DJ, Stanziano GJ, Saltzman DH. 2007. Increased incidence of gestational diabetes in women receiving prophylactic 17alpha-hydroxyprogesterone caproate for prevention of recurrent preterm delivery. Diabetes Care. 30:2277-2280. 
326

327

328

329

Reynolds MA, Schieve LA, Martin JA, Jeng G, Macaluso M. 2003. Trends in multiple births conceived using assisted reproductive technology, United States, 1997-2000. Pediatrics 111:1159-1162.

Saccone G, Zullo F, Roman A, Ward A, Maruotti G, Martinelli P, Berqhella V. 2017. Risk of spontaneous preterm birth in IVF-conceived twin pregnancies. J Matern Fetal Neonatal Med 21:1-8.

Sun L, Zou G, Wei X, Chen Y, Zhang J, Okun N, Duan T. 2016. Clinical outcomes after assisted reproductive technology in twin pregnancies: chorionicity-based comparison. Sci Rep 31:26869

Toulis KA, Goulis DG, Kolibianakis EM, Venetis CA, Tarlatzis BC, Papadimas I. 2009. Risk of gestational diabetes mellitus in women with polycystic ovary syndrome: a systematic review and a meta-analysis. Fertility and Sterility 92:667-677.

Vannuccini S, Clifton VL, Fraser IS, Taylor HS, Critchley H, Giudice LC, Petraqlia F. 2016. Infertility and reproductive disorders: impact of hormonal and inflammatory mechanisms on pregnancy outcome. Human Reproduction Update 22:104-115.

Vasario E, Borgarello V, Bossotti C, Libanori E, Biolcati M, Arduino S, Spinelli R, Delle Piane L, Revelli A, Todros T. 2010. IVF twins have similar obstetric and neonatal outcome as spontaneously conceived twins: a prospective follow-up study. Reproductive Biomedicine Online 21:422-428. 


\section{Figure 1 (on next page)}

A workflow to show inclusion criteria in the study

Figure $1 \mathrm{~A}$ workflow to show inclusion criteia 
487 dizygotic twin pregnancies were included

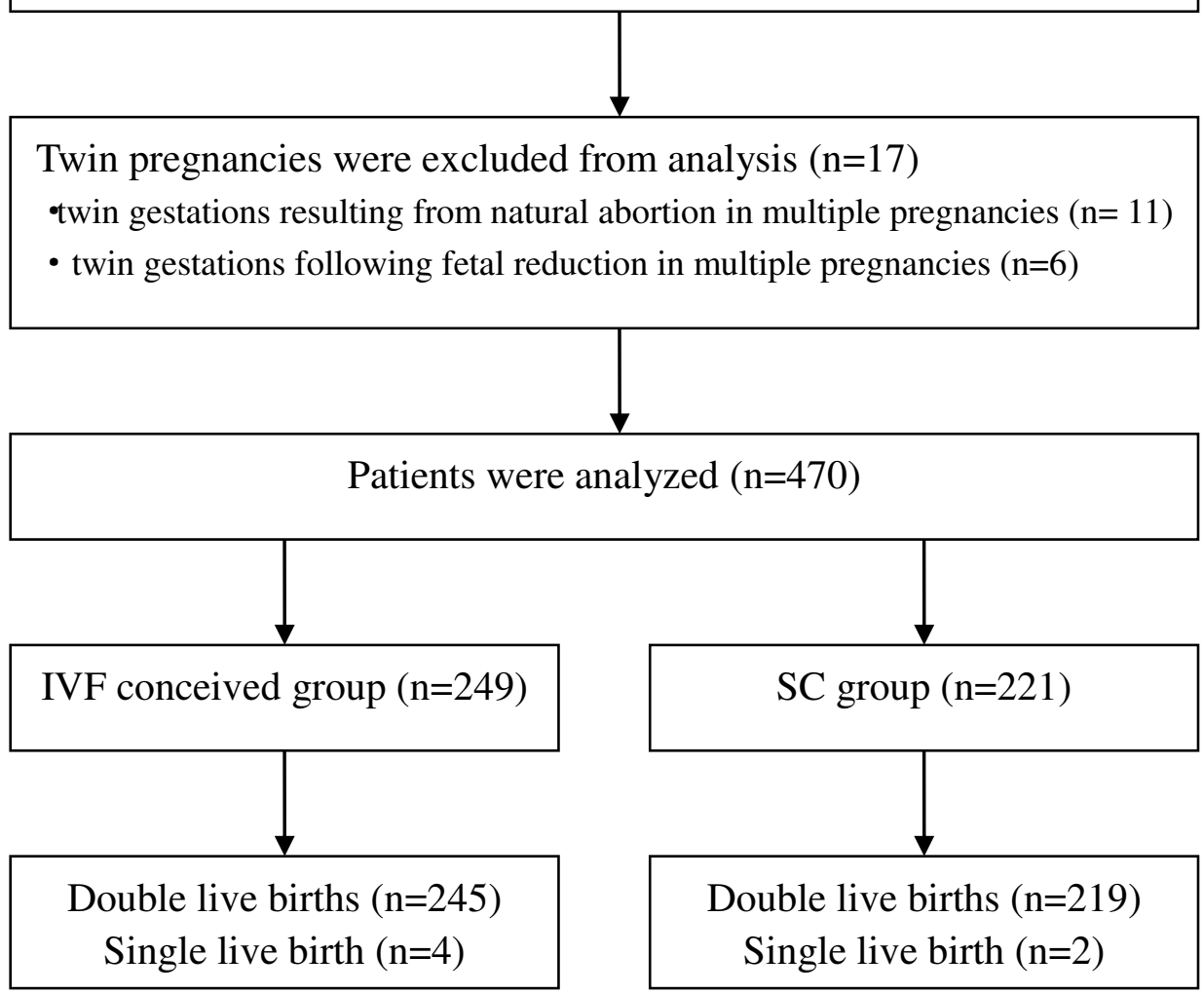

Figure 1. A workflow to show inclusion criteria in the study 
Table $\mathbf{1}$ (on next page)

Maternal characteristics and delivery modes in twin pregnancy 
1 Table 1 Maternal characteristics and delivery modes in twin pregnancy

\begin{tabular}{cccc}
\hline & SC $(\mathrm{n}=221)$ & IVF $(\mathrm{n}=249)$ & P value \\
\hline Age (y) & $28.44 \pm 4.27$ & $30.66 \pm 3.80$ & $<0.001$ \\
primiparity & $138(62.4)$ & $212(85.1)$ & $<0.001$ \\
gestational age (w) & $36.41 \pm 1.65$ & $36.07 \pm 1.71$ & $0.026^{*}$ \\
Cesarean section & $206(93.2)$ & $239(96.0)$ & 0.081 \\
First Cesarean section ${ }^{\#}$ & $24 / 82(29.3)$ & $10 / 36(27.8)$ & 0.527 \\
Length of hospitalization & $6.98 \pm 4.92$ & $7.34 \pm 4.99$ & 0.042 \\
(d) & & & 0.817 \\
\hline Birth weight $(\mathrm{kg})$ & $2490.57 \pm 432.37$ & $2484.05 \pm 428.03$ & \\
\hline
\end{tabular}

2 Notes: values are cases( \%) or mean \pm standard deviation; * the difference in mean gestational length attributes

3 to the difference in gestational length counting methods for the two groups; \# the comparison is restricted to 4 women with at least one previous delivery. 
Table 2 (on next page)

Maternal complications 
1 Table 2 Maternal complications

\begin{tabular}{|c|c|c|c|c|c|}
\hline complications & $\mathrm{SC}(\mathrm{n}=221)$ & $\operatorname{IVF}(n=249)$ & $\begin{array}{c}\text { Adjusted } \\
\text { OR }\end{array}$ & $\begin{array}{c}\text { Adjusted } 95 \% \\
\text { CI }\end{array}$ & $P$ value \\
\hline $\begin{array}{c}\text { gestational diabetes } \\
\text { mellitus }\end{array}$ & $32(14.5)$ & $56(22.5)$ & 1.24 & $0.74 \sim 2.10$ & 0.42 \\
\hline hypertensive disorder & $27(12.2)$ & $29(11.6)$ & 0.95 & $0.52 \sim 1.77$ & 0.88 \\
\hline $\begin{array}{c}\text { premature rupture of } \\
\text { membranes }\end{array}$ & $23(10.4)$ & $25(10.0)$ & 0.72 & $0.37 \sim 1.38$ & 0.32 \\
\hline maternal anemia & $110(49.8)$ & $141(56.6)$ & 1.51 & $1.01 \sim 2.26$ & 0.05 \\
\hline placenta previa & $5(2.3)$ & $12(4.8)$ & 2.08 & $0.66 \sim 6.61$ & 0.21 \\
\hline placental accreta & $7(3.2)$ & $13(5.2)$ & 1.33 & $0.48 \sim 3.69$ & 0.58 \\
\hline postpartum hemorrhage & $16(7.2)$ & $23(9.2)$ & 1.20 & $0.58 \sim 2.48$ & 0.63 \\
\hline $\begin{array}{l}\text { intrahepatic cholestasis of } \\
\text { pregnancies }\end{array}$ & $11(5)$ & $14(5.6)$ & 1.25 & $0.51 \sim 3.09$ & 0.63 \\
\hline polyhydramnios & $8(3.6)$ & $9(3.6)$ & 0.80 & $0.28 \sim 2.31$ & 0.68 \\
\hline
\end{tabular}

2 OR is the abbreviation of odds ratio, and $\mathrm{CI}$ is the abbreviation of confidence interval. 


\section{Table 3 (on next page)}

Neonatal outcomes 
1 Table 3 Neonatal outcomes

\begin{tabular}{|c|c|c|c|c|c|}
\hline & $\mathrm{SC}(\mathrm{n}=221)$ & $\operatorname{IVF}(n=248)$ & $\begin{array}{c}\text { Adjusted } \\
\text { OR }\end{array}$ & $\begin{array}{c}\text { Adjusted } 95 \% \\
\text { CI }\end{array}$ & $\mathrm{P}$ value \\
\hline Growth discordance & $15(6.8)$ & $14(5.7)$ & 0.76 & $0.34 \sim 1.70$ & 0.50 \\
\hline Low birth weight & $139(62.9)$ & $142(57.3)$ & 0.97 & $0.65 \sim 1.44$ & 0.86 \\
\hline Neonatal asphyxia & $16(7.2)$ & $14(5.7)$ & 0.90 & $0.58 \sim 1.42$ & 0.66 \\
\hline NICU admission & $54(24.4)$ & $64(25.8)$ & 0.80 & $0.36 \sim 1.76$ & 0.57 \\
\hline
\end{tabular}

2 Values are cases (\%) or mean (standard deviation); OR is the abbreviation of odds ratio, and CI is the 3 abbreviation of confidence interval. 
Table 4(on next page)

Stillbirth and malformation among the two groups 
1 Table 4 Stillbirth and malformation among the two groups

$\mathrm{SC}(\mathrm{n})$ $\operatorname{IVF}(n)$

Intrauterine fetal death (1) Intrauterine fetal death (4)

Cystic mass of chest wall (1)

Congenital heart malformation (2)

Congenital high jejunal atresia (1)

2 